\title{
ÁCIDO CANÁRICO (3,4-SECO DERIVADO DO LUPANO) EM PRÓPOLIS DO CEARÁ
}

\author{
Irineu L. de Albuquerque, Leonardo A. Alves, Telma L. G. Lemos* e Francisco J. Q. Monte \\ Departamento de Química Orgânica e Inorgânica, Universidade Federal do Ceará, CP 12200, 60451-970 Fortaleza - CE, Brasil \\ Raimundo Braz-Filho \\ Setor de Produtos Naturais, Centro de Ciências e Tecnologia, Universidade Estadual do Norte Fluminense, 28013-600 Campos \\ dos Goytacazes - RJ, Brasil
}

Recebido em 23/3/06; aceito em 6/9/06; publicado na web em 26/3/07

\begin{abstract}
CANARIC ACID (3,4-SECO-LUPANE DERIVATIVE) ISOLATED FROM PROPOLIS OF CEARÁ. Phytochemical investigation of a propolis sample from Alto Santo - Ceará, Brazil, allowed identification of triterpenes (lupeol, lupenone, germanicone, canaric acid) and flavonoids (quercetin, kaempferol and acacetin), which were identified by spectroscopic data (IR, MS, and NMR, including 2D techniques). This is the first report of canaric acid in propolis. Propolis extract and flavonoids showed antioxidant activity using a DPPH radical scavenging assay.
\end{abstract}

Keywords: propolis; flavonoids; canaric acid.

\section{INTRODUÇÃO}

A palavra própolis, de origem grega, significa guardar a cidade ${ }^{1}$. O material conhecido como própolis contém um conjunto de substâncias naturais armazenadas em produtos resinosos e balsâmicos. Tais substâncias são colhidas diretamente de diferentes partes e exsudados de plantas, que são acrescidas pelas produzidas através das secreções salivares das abelhas, cera e pólen para formação do produto final ${ }^{2}$. Assim, a própolis se apresenta freqüentemente como resinas constituídas por misturas complexas de substâncias naturais produzidas biogeneticamente pelas plantas e pelas abelhas, com a função de selar as colméias e proteger contra predadores.

Diversas atividades biológicas comprovadas já foram relatadas para própolis em diferentes regiões do planeta, tais como antiviral, antiinflamatória, antioxidante, anticancerígena. Tais resultados comprovam a consagração do uso popular e justificam o seu consumo no mundo inteiro como auxiliar no tratamento das mais diversas doenças, entre as quais problemas cardíacos, diabetes, câncer e processos inflamatórios ${ }^{3}$.

A composição química da própolis está diretamente relacionada com o tipo de vegetação da região onde é bioproduzida. Portanto, as substâncias naturais presentes se encontram diretamente relacionadas com a região de coleta da própolis, podendo conter mais de uma dezena de substâncias com diversas funções adicionais ainda desconhecidas pelo homem ${ }^{4,5}$.

Várias classes de substâncias naturais já foram isoladas e identificadas em própolis brasileira, destacando-se flavonóides ${ }^{6}$, flavanonas preniladas ${ }^{7}$, benzopirano ${ }^{8}$, benzofenona ${ }^{9}$, éster do ácido caféico $^{10}$, triterpenóides (cicloartano ${ }^{11}$, alcanoatos de lupeol ${ }^{12}$, ácido moronico ${ }^{13}$ e $3 \beta$-acetato de bauerenol ${ }^{14}$ ), derivados dos ácidos cinâmico e benzóico ${ }^{15}$ e epóxidos de naftoquinonas ${ }^{16}$.

Tais informações serviram como estímulo adicional para enfrentarmos o desafio investigativo da composição química da própolis do Ceará, já que contribui significativamente para a aquisição de conhecimento adicional da flora visitada pelas abelhas e para comparação fitoquímica. A amostra de própolis utilizada para investigação foi coletada no Município de Alto Santo-Ceará, região onde a vegetação

*e-mail: tlemos@dqoi.ufc.br predominante é a "caatinga". Assim, do extrato etanólico foram isolados e caracterizados os triterpenos conhecidos por ácido canárico (ácido 3,4-seco derivado do lupano (1), lupeol (2), lupenona (3) e germanicona (4) e os flavonóides quercetina (5), canferol (6) e acacetina (7). O ácido canárico (1) foi isolado anteriormente da resina de Canarium muelleri $^{17}$ e, mais recentemente, de Rugea jasminoides ${ }^{18}$, sendo este o primeiro registro desta substância em própolis.

\section{PARTE EXPERIMENTAL}

\section{Procedimentos experimentais gerais}

Nas análises cromatográficas de adsorção em coluna foi utilizada gel de sílica. O comprimento e o diâmetro das colunas foram estabelecidos com base nas quantidades das amostras e de gel de sílica utilizadas. Nas cromatografias de camada delgada (CCD) foram usadas cromatoplacas de sílica gel $\mathrm{GF}_{254}$. Os espectros de absorção na região do infravermelho (IV) foram obtidos em espectrômetro Perkin Elmer, modelo FT-IR Spectrum 1000, usando-se pastilhas de $\mathrm{KBr}$. Os espectros de $\mathrm{RMN}^{1} \mathrm{H}$ e ${ }^{13} \mathrm{C}$ foram registrados em espectrômetro modelo Avance DRX-500, usando-se $\mathrm{CDCl}_{3}$ como solvente e TMS como padrão interno. O espectro de massa foi obtido em espectrômetro Shimadzu QP5050A, operando em $70 \mathrm{eV}$. As leituras de Ultra-violeta foram feitas em equipamento modelo U-2000, Hitachi.

\section{Coleta da amostra}

A amostra de própolis usada para estudo foi produzida pela espécie Apis mellifera, coletada no município de Alto Santo - Ceará, Brasil, e mantida sob refrigeração.

\section{Extração e isolamento}

A amostra de própolis (750 g) foi extraída exaustivamente com EtOH. O resíduo obtido após a destilação do solvente (EBEP, 170 g) foi cromatografado em coluna (CC) filtrante de gel de sílica, sendo utilizados como eluentes, solventes em ordem crescente de polaridade: hexano, $\mathrm{CHCl}_{3}$, AcOEt e metanol. A fração $\mathrm{CHCl}_{3}$ foi subme- 
tida a sucessivas $\mathrm{CC}$ em gel de sílica, tendo como eluentes misturas binárias de Hex:AcOEt em ordem crescente de polaridade. A análise das frações através de CCD permitiu reuni-las em grupos frações e o isolamento subseqüente de $\mathbf{1}(45 \mathrm{mg})$, lupeol $\mathbf{2}(500 \mathrm{mg})$, lupenona $\mathbf{3}$ $(45 \mathrm{mg})$ e germanicona $4(30 \mathrm{mg})$. Cromatografias sucessivas da fração AcOEt, com mistura binária Hex:AcOEt em ordem crescente de polaridade, possibilitaram o isolamento de quercetina $\mathbf{5}(120 \mathrm{mg})$, canferol 6 (120 mg) e acacetina 7 (60 mg) (Figura 1).
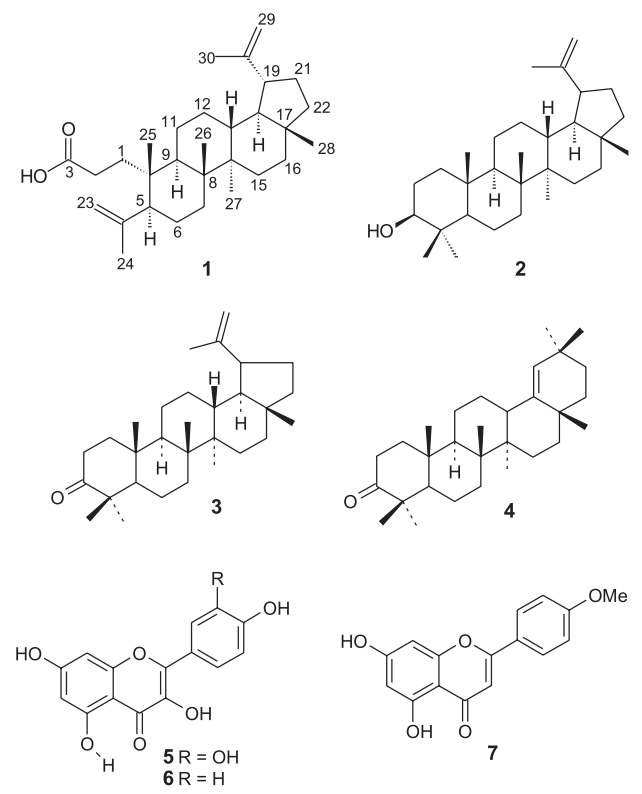

Figura 1. Substâncias de 1-7 isoladas de amostra de própolis de Alto Santo - Ceará

\section{Atividade antioxidante pelo método de seqüestro de radical DPPH}

Para avaliação da atividade antioxidante foi utilizado o método de seqüestro de radical DPPH e comparação com padrões positivos, usando a metodologia proposta por Hegazy e El-Hady ${ }^{19}$. Amostras do extrato etanólico de própolis (Extpr) e das substâncias 1-7 nas concentrações de 1,$00 ; 0,05$ e $0,025 \mathrm{~g} / \mathrm{L}$, foram dissolvidas em $1,0 \mathrm{~mL}$ de etanol e adicionadas a uma solução etanólica de DPPH $(1,0 \mathrm{~mL})$, na concentração de $60 \mu \mathrm{M}$. Foram realizadas medidas de absorbância na faixa de $520 \mathrm{~nm}$ em espectrofotômetro de UV, no tempo inicial e após 30 min. A percentagem de inibição foi obtida por comparação da absorção da solução contendo amostra, em relação a uma solução controle de DPPH sem amostra.

Os resultados mostrados na Tabela 2 representam a média aritmética de 3 leituras. Como padrões positivos de referência utilizaram-se Trolox e BHT.

\section{Propriedades físicas}

Ácido canárico (1) - sólido, p.f. $208-210{ }^{\circ} \mathrm{C},[\alpha]_{\mathrm{D}}^{25}+31^{\circ}[1,0$; $\mathrm{CHCl}_{3}$; I.V. $\left(\mathrm{KBr}, v_{\max } \mathrm{cm}^{-1}\right)-1641,1703,3487$; EM-IE, $70 \mathrm{eV}$, $m / z$ 440. RMN ${ }^{13} \mathrm{Ce}^{1} \mathrm{H}$, ver Tabela 1 .

\section{RESULTADOS E DISCUSSÃO}

Os triterpenos lupeol (2), lupenona (3), germanicona (4) e os flavonoides quercetina (5), canferol (6) e acacetina (7) foram identificados através de métodos espectroscópicos envolvendo a comparação com dados da literatura ${ }^{20-24}$.
O triterpeno 1 apresentou no espectro de IV absorções referentes à presença de ligação dupla $\mathrm{C}=\mathrm{C}\left(v_{\max } 1641 \mathrm{~cm}^{-1}\right) \mathrm{e}$ de grupos carboxila $\left(v_{\max } 1703 \mathrm{~cm}^{-1}\right)$ e hidroxílico $\left(v_{\max } 3487 \mathrm{~cm}^{-1}\right)$. O espectro de massas obtido por impacto eletrônico (EM-IE) revelou o pico correspondente ao íon molecular em $\mathrm{m} / \mathrm{z}$ 440, compatível com a formula molecular $\mathrm{C}_{30} \mathrm{H}_{48} \mathrm{O}_{2}$.

A análise comparativa dos espectros de $\operatorname{RMN}{ }^{13} \mathrm{C}\left\{{ }^{1} \mathrm{H}\right\}$ e RMN ${ }^{13} \mathrm{C}$-DEPT $135^{\circ}$ de $\mathbf{1}$ permitiu identificar 30 sinais (Tabela 1) correspondentes a sete átomos de carbono não hidrogenados [três $\mathrm{sp}^{2}: \delta_{\mathrm{C}} 181,00(\mathrm{COOH}) ; 152,42 ; 149,18$; quatro $\mathrm{sp}^{3}: \delta_{\mathrm{C}} 44,83 ; 44,59$; $42,13$ e 40,79$]$, cinco metínicos (todos $\mathrm{sp}^{3}: \delta_{\mathrm{C}} 51,97 ; 49,82 ; 49,56$; $42,32$ e 39,70$)$, doze metilênicos [dois sp $2: \delta_{C} 114,99\left(\mathrm{CH}_{2}-24\right)$ e $111,06\left(\mathrm{CH}_{2}-29\right)$; dez sp $: \delta_{\mathrm{C}} 41,56 ; 37,07 ; 35,48 ; 34,34 ; 31,40$; $29,77 ; 29,05 ; 26,61 ; 26,27$ e 23,06$]$ e seis metílicos $\left(\delta_{\mathrm{C}} 24,81 ; 21,69\right.$; $20,88 ; 19,59 ; 17,56$ e 16,06$)$ e classificar como um triterpeno tetracíclico contendo um grupo carbonila de ácido $\left(\delta_{\mathrm{C}} 180,00\right) \mathrm{e}$ duas ligações olefínicas terminais $\left[\left(\mathrm{C}=\mathrm{CH}_{2}\right)_{2}: \delta_{\mathrm{C}} 149,18(\mathrm{C}-4)\right.$ e $114,99\left(\mathrm{CH}_{2}-24\right)$; e $\delta_{\mathrm{C}} 152,42(\mathrm{C}-20)$ e $\left.111,06(\mathrm{C}-29)\right]$.

A análise do espectro de $\mathrm{RMN}^{1} \mathrm{H}$ confirmou a presença de quatro átomos de hidrogênio olefínicos em duplas terminais pelos sinais simples em $\delta_{\mathrm{H}} 4,66$ e 4,85 (2H-24) e em $\delta_{\mathrm{H}} 4,58$ e 4,70 $(2 \mathrm{H}-$ 29). Os deslocamentos químicos dos sinais simples em $\delta_{\mathrm{H}} 1,73 \mathrm{e}$ 1,70 foram atribuídos aos dois grupos metílicos ligados a átomos de carbono $\mathrm{sp}^{2}$ (Tabela 1).

Tabela 1. Dados de $\mathrm{RMN}^{1} \mathrm{H}$ e ${ }^{13} \mathrm{C}$ de $1\left[\mathrm{CDCl}_{3}\right]$, incluindo-se resultados de correlação heteronuclear ${ }^{1} \mathrm{H}^{-13} \mathrm{C}-\mathrm{COSY}-{ }^{1} \mathrm{~J}_{\mathrm{CH}}$ (HMQC) e ${ }^{1} \mathrm{H}^{-13} \mathrm{C}-\mathrm{COSY} \mathrm{H}^{-} \mathrm{J}_{\mathrm{CH}}(\mathrm{HMBC})$. Deslocamentos químicos em $\delta_{\mathrm{C}}$ e $\delta_{\mathrm{H}}$ (ppm) e constantes de acoplamento ( $J$, entre parênteses) em $\mathrm{Hz}$

\begin{tabular}{|c|c|c|c|c|}
\hline \multicolumn{3}{|c|}{ HMQC } & \multicolumn{2}{|c|}{ НMBC } \\
\hline & $\delta_{\mathrm{C}}$ & $\delta_{\mathrm{H}}$ & ${ }^{2} \mathrm{~J}_{\mathrm{CH}}$ & ${ }^{3} \mathbf{J}_{\mathrm{CH}}$ \\
\hline 1 & 35,48 & $1,62(\mathrm{~m}) ; 2,33(\mathrm{~m})$ & $2 \mathrm{H}-2$ & $3 \mathrm{H}-25$ \\
\hline 2 & 29,77 & $2,20(\mathrm{~m}) ; 2,35(\mathrm{~m})$ & - & \\
\hline 3 & 181,00 & & $2 \mathrm{H}-2$ & \\
\hline 4 & 149,18 & & $3 \mathrm{H}-23$ & \\
\hline 5 & 51,97 & $1,94(\mathrm{~d}, 10,5)$ & & $\begin{array}{l}\mathrm{H}-1 \mathrm{~b} ; 2 \mathrm{H}-24 ; \\
3 \mathrm{H}-23 ; 3 \mathrm{H}-25\end{array}$ \\
\hline 6 & 26,27 & $1,35(\mathrm{~m}) ; 1,65(\mathrm{~m})$ & & \\
\hline 7 & 34,34 & $1,48-1,35(\mathrm{~m})$ & & $3 \mathrm{H}-26$ \\
\hline 8 & 42,13 & & $3 \mathrm{H}-26$ & $3 \mathrm{H}-27$ \\
\hline 9 & 42,32 & $1,41(\mathrm{~m})$ & & $3 \mathrm{H}-25 ; 3 \mathrm{H}-26$ \\
\hline 10 & 40,79 & & $\mathrm{H}-5 ; 3 \mathrm{H}-25$ & \\
\hline 11 & 23,06 & $1,24(\mathrm{~m}) ; 1,30(\mathrm{~m})$ & & \\
\hline 12 & 26,61 & $1,05(\mathrm{~m}) ; 1,70(\mathrm{~m})$ & & \\
\hline 13 & 39,70 & $1,68(\mathrm{~m})$ & & $3 \mathrm{H}-27$ \\
\hline 14 & 44,83 & & $3 \mathrm{H}-27$ & $3 \mathrm{H}-26$ \\
\hline 15 & 29,05 & $1,05(\mathrm{~m}) ; 1,70(\mathrm{~m})$ & & $3 \mathrm{H}-27$ \\
\hline 16 & 37,07 & $1,40(\mathrm{~m}) ; 1,50(\mathrm{~m})$ & & $3 \mathrm{H}-28$ \\
\hline 17 & 44,59 & & $3 \mathrm{H}-28$ & \\
\hline 18 & 49,82 & $1,40(\mathrm{~m})$ & H-19 & $3 \mathrm{H}-28$ \\
\hline 19 & 49,56 & $2,37(\mathrm{~m})$ & & $\mathrm{H}-29 \mathrm{a}$ \\
\hline 20 & 152,42 & & H-19; 3Н-30 & \\
\hline 21 & 31,40 & $1,35(\mathrm{~m}) ; 1,90(\mathrm{~m})$ & & \\
\hline 22 & 41,56 & $1,20(\mathrm{~m}) ; 1,38(\mathrm{~m})$ & & $3 \mathrm{H}-28$ \\
\hline 23 & 114,99 & $4,66(\mathrm{~s}) ; 4,85(\mathrm{~s})$ & & $3 \mathrm{H}-23$ \\
\hline 24 & 21,69 & $1,73(\mathrm{~s})$ & & \\
\hline 25 & 24,81 & $0,85(\mathrm{~s})$ & & \\
\hline 26 & 17,56 & $1,08(\mathrm{~s})$ & & - \\
\hline 27 & 16,06 & 0,97 (s) & & - \\
\hline 28 & 19,59 & $0,80(\mathrm{~s})$ & & - \\
\hline 29 & 111,06 & $4,58(\mathrm{~s}) ; 4,70(\mathrm{~s})$ & & H-19; 3H-30 \\
\hline 30 & 20,88 & $1,70(\mathrm{~s})$ & & Н-19; $2 \mathrm{H}-29$ \\
\hline
\end{tabular}


Estas deduções e as informações adicionais obtidas pela interpretação dos espectros de massas (Figura 2) e de RMN de ${ }^{1} \mathrm{H} \mathrm{e}{ }^{13} \mathrm{C}$ (500 e $125 \mathrm{MHz}$, em $\mathrm{CDCl}_{3}$ ), (DEPT $135^{\circ}$ ) e $2 \mathrm{D}$ de correlação homonuclear (COSY) e heteronuclear (HMQC e HMBC), envolvendo inclusive a comparação com dados da literatura ${ }^{17,18}$, permitiram caracterizar a estrutura do triterpeno ácido 3,4-seco-derivado do lupano (ácido canárico, 1) e estabelecer a atribuição inequívoca dos deslocamentos químicos dos átomos de hidrogênio e carbono (Tabela 1). Análise detalhada do espectro de HMBC, mostrou correlações entre $\mathrm{C}-5\left(\delta_{\mathrm{C}} 51,97\right)$ com $2 \mathrm{H}-24,3 \mathrm{H}-23,3 \mathrm{H}-25$ e H-1b, e do $\mathrm{C}-9\left(\delta_{\mathrm{C}} 42,32\right)$ com $3 \mathrm{H}-25$, e $3 \mathrm{H}-26$.

A estereoquímica relativa de $\mathbf{1}$ foi deduzida com base nos dados de interação dipolar-dipolar revelada pelo espectro $2 \mathrm{D}{ }^{1} \mathrm{H}-{ }^{1} \mathrm{H}-$ NOESY. Nesse espectro foi observado forte efeito NOE entre os átomos de hidrogênio: $\mathrm{H}-9\left(\delta_{\mathrm{H}}, 1,41\right)$ e $3 \mathrm{H}-27\left(\delta_{\mathrm{H}} 0,97\right)$ e $\mathrm{H}-5\left(\delta_{\mathrm{H}}\right.$ $1,94) ; 3 \mathrm{H}-23\left(\delta_{\mathrm{H}} 1,73\right)$ e H-24a $\left(\delta_{\mathrm{H}} 4,85\right) ; \mathrm{H}-29 \mathrm{a}\left(\delta_{\mathrm{H}} 4,70\right)$ e H-19 $\left(\delta_{\mathrm{H}} 2,37\right) ; \mathrm{H}-5\left(\delta_{\mathrm{H}}, 94\right)$ e H-24b $\left(\delta_{\mathrm{H}} 4,66\right) ; 3 \mathrm{H}-25\left(\delta_{\mathrm{H}} 0,85\right) \mathrm{com}$

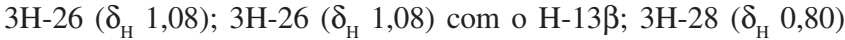
com $3 \mathrm{H}-26$ e H-13b; 3H-27 $\left(\delta_{\mathrm{H}} 0,97\right)$ com H-18 $\left(\delta_{\mathrm{H}} 1,40\right) ; 3 \mathrm{H}-30$ $\left(\delta_{\mathrm{H}} 1,70\right)$ com H-29b $\left(\delta_{\mathrm{H}} 4,58\right)$.

Apesar de ser uma substância descrita anteriormente em espécies vegetais, este é o primeiro registro deste triterpeno em própolis. Uma proposta de fragmentação de $\mathbf{1}$ no espectrômetro de massas encontra-se resumida na Figura 2. Os fragmentos $\mathrm{m} / \mathrm{z} 189$ (62\%) e $\mathrm{m} / \mathrm{z}, 203$ (59\%), picos importantes no espectro, foram originados por quebra das ligações $\mathrm{C}_{8}-\mathrm{C}_{14} / \mathrm{C}_{12}-\mathrm{C}_{13}$ e $\mathrm{C}_{8}-\mathrm{C}_{14} / \mathrm{C}_{9}-\mathrm{C}_{11}$ respectivamente, de acordo com a literatura ${ }^{25}$.

Os compostos 2 e 3 apresentaram dados de RMN ${ }^{13} \mathrm{C}$ e ${ }^{1} \mathrm{H}$ compatíveis com triterpenos com esqueleto tipo lupano: $\delta_{\mathrm{H}} 4.54$ (dl, $1 \mathrm{H}), 4,67(\mathrm{dl}, 1 \mathrm{H})$ e $1,68(\mathrm{sl}, 3 \mathrm{H}), \delta_{\mathrm{C}} 151,00(\mathrm{C})$ e $109,59\left(\mathrm{CH}_{2}\right)$. Todas estas absorções caracterizam o grupo isoprenila presente nesse esqueleto. Para o composto 2 observou-se absorção em $\delta_{C}$ $79,08(\mathrm{CH})$ relativa de $\mathrm{C}-3 \mathrm{em}$ triterpenos $3 \beta-\mathrm{OH}$, enquanto que o composto 3, apresentou uma carbonila em $\delta_{\mathrm{C}} 218,20$. Os dados apresentados são compatíveis com lupeol e lupenona ${ }^{21-23}$.

$\mathrm{O}$ espectro de $\mathrm{RMN}{ }^{13} \mathrm{C}$ do composto 4 exibiu sinais em $\delta_{\mathrm{C}}$ $142,77(\mathrm{C})$ e 130,08 (CH) indicativos de ligação olefínica de triterpeno oleanano. Em adição, um sinal em $\delta_{\mathrm{C}} 218,11(\mathrm{C}=\mathrm{O})$ e comparação com dados de RMN ${ }^{1} \mathrm{H}$ e ${ }^{13} \mathrm{C}$, IV com aqueles registrados na literatura permitiram caracterizar 4 como o triterpeno olean-18en-3-ona, conhecido como germanicona ${ }^{21-23}$.

Os compostos 5, 6, e 7 exibiram nos espectros de RMN ${ }^{13} \mathrm{C}$ quinze sinais na região de carbonos $\mathrm{sp}^{2}$, compatíveis com a unidade $\mathrm{C}_{6}-\mathrm{C}_{3}-\mathrm{C}_{6}$ característica de flavonóides. A mistura binária constituída de 5 e 6, permitiu identificar em 5 a presença de cinco carbonos não hidrogenados, oxigenados em (C-3, C-5, C-7, C-3', e C-4'), referente a carbonos contendo grupos hidroxila e apenas quatro absorções de carbonos oxigenados em 6 (C-3, C-5, C-7, e C-4'). Análise detalhada dos espectros de $\mathrm{RMN}{ }^{13} \mathrm{C}$ e ${ }^{1} \mathrm{H}$, de 5 permitiu sua identificação como sendo 3,5,7,3'4'-pentaidroxiflavone, enquanto o composto 6 apresentou somente quatro carbonos $\mathrm{sp}^{2}$ oxigenados, sendo identificado como 3,5,7,4'-tetraidroxiflavone. $\mathrm{O}$ espectro de RMN ${ }^{13} \mathrm{C}$ do composto 7 apresentou três sinais de carbonos $\mathrm{sp}^{2}$ oxigenados, dois contendo grupos hidroxila e um com metoxila em $\left(\delta_{\mathrm{C}} 58,00\right)$ na posição $\mathrm{C}-4$ ', dedução corroborada através do espectro de $\mathrm{RMN}{ }^{1} \mathrm{H}$ pelos sinais de hidrogênios aromáticos de um sistema para-substituído, e possibilitaram sua identificação como sendo 5,7-diidroxi-4' -metoxiflavone. Comparação dos dados espectroscópicos obtidos para os compostos 5-7 com os registrados na literatura permitiu a identificação destes constituintes como sendo quercetina, canferol e acacetina, respectivamente ${ }^{24}$.

O extrato etanólico de própolis (Extpr) e as substâncias 1-7 foram submetidas a teste de atividade antioxidante, utilizando-se o

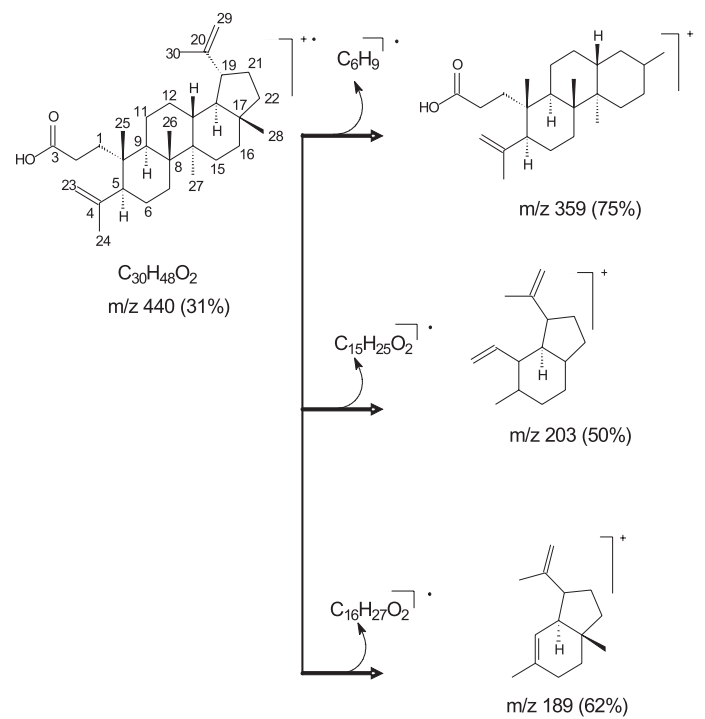

Figura 2. Fragmentos propostos para justificar os picos principais observados no espectro de massas (EM) do triterpeno 1. Reproduzida da ref. 25, com permissão da ACS

método de seqüestro de radicais DPPH ${ }^{16}$. Através dessa metodologia foi observada atividade significativa para os flavonóides quercetina 5, canferol 6 e acacetina 7 , que apresentaram redução da formação de radicais na concentração de 1,00 mg de 70, 68 e 53\% e na concentração de 0,25 mg, inibição de 70, 59 e 19\%, respectivamente. Estes dados estão compatíveis com as estruturas, verificando-se que os flavonóides com maior número de hidroxilas, como a quercetina e canferol, apresentaram maior percentagem de inibição. Para o ácido canárico observou-se fraca atividade de inibição, com redução de apenas $26 \%$ na concentração de $1,00 \mathrm{mg} / \mathrm{mL}$ e ausência de atividade para os triterpenos 2-4, cujos resultados são mostrados na Tabela 2.

Tabela 2. Atividade antioxidante por seqüestro de radical DPPH de extrato etanólico de própolis e das substâncias 1, 5-7

\begin{tabular}{lccc}
\hline \multirow{2}{*}{ Substâncias } & \multicolumn{3}{c}{ Concentração $(\mathrm{mg} / \mathrm{mL})$} \\
& 1,00 & 0,05 & 0,025 \\
\hline Extpr & \multicolumn{3}{c}{} \\
$\mathbf{1}$ & 66 & 50 & 45 \\
$\mathbf{5}$ & 26 & 25 & 22 \\
$\mathbf{6}$ & 70 & 70 & 70 \\
$\mathbf{7}$ & 68 & 60 & 59 \\
BHT & 53 & 29 & 19 \\
Trolox & 100 & 98 & 97 \\
\hline
\end{tabular}

\section{AGRADECIMENTOS}

Aos órgãos de fomento à pesquisa CNPq, CAPES, FUNCAP e FAPERJ pelas bolsas e auxílios concedidos e ao CENAUREMNUFC pelas oportunidades disponibilizadas para obtenção dos espectros de RMN.

\section{REFERENCIAS}

1. Ghisabert, E. L.; Bee World 1979, 60, 59.

2. Marcucci, M. C.; Apidologie 1995, 26, 83.

3. Marcucci, M. C.; Quím. Nova 1996, 19, 529; Hegazi, A. G.; Bee Informed 1998, 5, 22; Matsushige, K.; Basnet, P.; Kadota, S.; Namba, T.; J. Traditional Medicines 1996, 13, 217. 
4. Custódio, A. R.; Ferreira, M. M. C.; Negri, G.; Salatino, A.; J. Braz. Chem. Soc. 2003, 14, 354

5. Menezes, H.; Arq. Ins. Biol. 2005, 72, 405.

6. Chen, C. N.; Wu, C. L.; Shy, H. S.; Lin, J. K.; J. Nat. Prod. 2003, 66, 503.

7. Matsuya, M.; Saito, M.; Matsuyuki, Y.; Morikawa, J.; Z. Naturforsch., C: J. Biosci. 1998, 53, 1037.

8. Rubio, O. C.; Cuellar, A. C.; Rojas, N.; Castro, H. V.; Rastrelli, L.; Aquino, R.; J. Nat. Prod. 1999, 62, 1013.

9. Banskota, A. H.; Tezuka, Y.; Midorikawa, K.; Matsushige, K.; Kadota, S.; J. Nat. Prod. 2000, 63, 1277.

10. Nagaoka, T.; Banskota, A. H.; Tezuka, Y.; Midorikawa, K.; Matsushige, K.; Kadota, S.; Biol. Pharm. Bull. 2003, 26, 487.

11. Silva, M. S. S.; Citó, A .M. G. L.; Chaves, M. H.; Dantas, J. A.; Quim. Nova 2005, 28, 801

12. Pereira, A. S.; Nascimento, E. A.; Neto, F. R. A.; Z. Naturforsch., C: J. Biosci. 2002, 57, 721 .

13. Ito, J.; Chang, F. R.; Wang, H. K.; Park, Y. K.; Ikegaki, M.; Kilgore, N.; Lee, K. H.; J. Nat. Prod. 2001, 64, 1278.

14. Teixeira, E. W.; Message, D.; Negri, G.; Salatino, A.; Quim. Nova 2006, 29, 245.

15. Salatino, A.; Teixeira, E. W.; Negri, G.; Message, D.; Evid. Based Complement. Alternat. Med. 2005, 2, 33 .
16. Trusheva, B.; Popova, M.; Bankova, V.; Simova, S.; Marcucci, M. C. Miorin, P. L.; Pasin, F. R.; Tsvetkova, I.; Evid. Based Complement. Alternat. Med. 2006, 3, 249.

17. Carman, R. M.; Cowley, D. E.; Tetrahedron Lett. 1964, 12, 627.

18. Lopes, M. N.; Mazza, F. C.; Young, M. C. M.; Bolzani, V. S.; J. Braz. Chem. Soc. 1999, 10, 237.

19. Hegazi, A. G.; El Hady, F. K. A.; Z. Naturforsch., C: J. Biosci. 2002, 57, 395.

20. Patra, A.; Mukhopadhyay, A.; Mitra, A. K.; Org. Magn. Reson. 1981, 17, 166; Pinheiro, M. L. B.; da Rocha, A. F. I.; Fernandes, M. A.; Monte, F. J. Q.; Villar, J. D. F.; Cruz, E. R.; Quim. Nova 2004, 27, 188.

21. Roshan, C. C.; Sotheeswaram, S.; Uvais, M.; Sultanbawa, S.; Ternai, B.; Org. Magn. Reson. 1980, 14, 462.

22. Olea, R. S. G.; Roque, N. F.; Quim. Nova 1990, 13, 278.

23. Ahmad, V. U.; Arman, A. U.; Handbook of natural products data: Pentacyclic triterpenoids, Elsevier Science: Netherland, 1994.

24. Agraval, P. K.; Carbon-13 NMR of flavonoids, Elsevier: Netherlands, 1989; Studies in Organic Chemistry, 1989, vol. 39.

25. Dallavalle, S.; Jayasinghe, L.; Kumarihamy, B. M. M.; Merlini, L.; Musso, L.; Scaglioni, L.; J. Nat. Prod. 2004, 67, 911. 